\title{
Utilizing Social Norms Marketing to Address E-Cigarette Use on College Campuses
}

\author{
Kristen Scholly ${ }^{1,}$, Lynn Garcia ${ }^{2}$, Stormy Dodge ${ }^{1}$, and Pallav Pokhrel $^{3}$ \\ ${ }^{1}$ University Health Services Mānoa, Health Promotion Program, University of Hawaii at Mānoa, 1710 \\ East-West Rd, Honolulu, HI 96822, USA \\ ${ }^{2}$ Kapiolani Medical Center for Women and Children, 1319 Punahou St, Honolulu, HI 96826, Hawaii, USA \\ ${ }^{3}$ University of Hawaii Cancer Center, Cancer Prevention Program, 701 Ilalo St, Honolulu, HI 96813, \\ Hawaii, USA
}

\begin{abstract}
E-cigarette use is a serious health concern among adolescents and young adults. This study identifies factors associated with e-cigarette use in an effort to better understand what impacts college students' decisions to use this tobacco product. This study utilizes data from a survey conducted at a research institution and two community colleges located in Hawaii. A multivariate logistic regression analysis identified key risk factors associated with college student e-cigarette use. Independent factors associated with e-cigarette use included use of traditional cigarettes and having one or more family members or friends who currently used e-cigarettes. Exposure to online e-cigarette information was also associated with increased odds of e-cigarette use. The study findings have important implications for e-cigarette control among adolescents and young adults. Service learning students can take an active role in developing social norms marketing campaigns designed to communicate the risks of e-cigarette use and counter marketing ploys that foster unsubstantiated beliefs regarding the benefits of e-cigarette use.
\end{abstract}

Keywords: College health, e-cigarette use, service learning, social norms marketing.

\section{Introduction}

The use of electronic cigarettes or e-cigarettes, is a growing global trend especially among adolescents and young adults. Although there is a wide range of e-cigarettes, most share a common basic design. E-cigarettes are battery-operated devices which may or may not look similar to traditional cigarettes and deliver an aerosol which is often flavored and contains nicotine [1]. There is increasing evidence that e-cigarette aerosol contains carcinogens and other toxins that can lead to negative health consequences [1]. For example, the aerosol contains harmful chemicals such as formaldehyde, lead, nickel and acetaldehyde all of which can cause cancer, birth defects and other reproductive harm $[2,3]$.

Adolescents and young adults are often misinformed about the negative health effects of ecigarette use. E-cigarette products are aggressively marketed as highly palatable, less harmful and readily accessible alternatives to other tobacco products $[3,4]$. According to a 2016 study, $79 \%$ of college students surveyed saw e-cigarettes as posing little to moderate harm and $71 \%$

\footnotetext{
${ }^{*}$ Corresponding author: kristen@hawaii.edu
} 
saw e-cigarettes as not addictive or only somewhat addictive [5]. Results also indicated that individuals who believe e-cigarettes present a low degree of harmfulness and addictiveness may be more inclined to use them [5]. In addition, dual users of both traditional and e-cigarettes were 3.5 times more likely than nonusers to perceive e-cigarettes as posing little to no harm [5].

Another concern is the influence of family or friends regarding adolescent and young adult e-cigarette use. Previous research has shown that having friends and family members who smoke impacts young people's tendency to smoke e-cigarettes. For example, in a study of adolescents with family members who smoke traditional cigarettes, individuals were 3.4 times more likely to use e-cigarettes when compared to those with no one in their household who smoked [6].

E-cigarettes not only have a strong advertising presence on television and in print advertisements, but also on the Internet, which has become a primary means of communication for adolescents and young adults [7,8]. All the major tobacco companies now own e-cigarette brands and the same advertising tactics used to lure young people to traditional cigarettes are being used to market e-cigarettes $[9,10]$. As a result, it is important to understand the impact that online and TV exposure to e-cigarettes have on subsequent college student e-cigarette use.

\section{Methods}

\subsection{Participants}

The study participants included 307 undergraduate students recruited from a university and two community colleges. The human subject protection protocol for this research was approved by the Western Institutional Review Board (Protocol \#20130949). Data were collected on participants' age, gender, and ethnicity. The mean age of study participants was $23.5 \mathrm{yr}$. More women than men participated in the study, which was expected, as women make up $55 \%$ of the student population in the university system where the study was conducted. Participants' ethnic distribution was similar to the ethnic distribution of the university system's student population except that the proportions of Filipinos (30\%) and Native Hawaiian/Pacific Islanders (NH/PI) $(13 \%)$ in the sample were higher and lower than the proportions of Filipino $(9 \%)$ and NH/PI $(17 \%)$ students in the university system, respectively.

\subsection{Recruitment and data collection}

Data were collected online. Participants were recruited using on-campus advertisements at a 4 yr college/university and two community or 2 yr colleges. The study was advertised using flyers, ads in student newspapers, classroom and dorm announcements, and student life e-mail bulletins. Each type of advertisement guided students to the study webpage by providing a brief description of the study and a web-link to study webpage. The study webpage directed students who consented to participate in the screener survey which collected information on potential participants' age, gender, lifetime and past $30 \mathrm{~d}$ cigarette smoking and college e-mail address. The students who met the first set of eligibility criteria were invited via e-mail to participate in the main study survey. The e-mail invitation included a web-link, unique for each participant, which sent students to the study survey. Students who met the initial screening criteria were further screened to recruit: i) approximately equal proportions of cigarette never-smokers, experimenters or ex-smokers, and current smokers (daily and occasional); and ii) an adequate proportion of men. Of the 326 students who were invited via e-mail to participate in the main study survey, 307 (94\%) completed the survey. 


\subsection{Information about e-cigarettes through online and TV sources}

Participants were asked "From where do you get most of your information about e-cigarettes?" Response options included, among others, "Advertisements on the internet," "Advertisements on TV," "The internet but from sources other than advertisements," and "TV programs but from sources other than advertisements." In addition, participants were asked, "How often have you felt curious about e-cigarettes and looked them up online?" Response was assessed on a 4-point scale ranging from "Never" to "Often."

\subsection{Family and friend e-cigarette use}

Family e-cigarette use was measured by a single question: "How many individuals in your immediate family (e.g., parents, siblings) use e-cigarettes?" Friend e-cigarette use was also measured with a single question: "How many of your close friends use e-cigarettes?"

\subsection{Cigarette smoking}

Cigarette smoking was assessed in terms of lifetime cigarette use, past $30 \mathrm{~d}$ cigarette use, and self-reported current smoking status. Lifetime cigarette smoking was determined based on a single item, "How many cigarettes have you smoked in your entire life?" (None, 1 to 100, $>100$ ). Past $30 \mathrm{~d}$ cigarette use was assessed by asking, "Within the last $30 \mathrm{~d}$, on how many days did you use cigarettes?" Response options included "Never used," "Have used, but not in last 30 d," "Have used within the last $1 \mathrm{~d}$ to $29 \mathrm{~d}$," and "Used daily." Current daily smoking was determined based on the following question: "How do you describe your cigarette smoking behavior?" Response options included "I do not smoke," "I smoke sometimes," and "I smoke every day." Smoker status was determined using all three measures. Students who reported having never smoking on all three measures were labeled "Never Smokers." Students who reported having used one or more cigarettes in the past and self-identified one as a current smoker was labeled "Current Smoker." And students who reported having used any cigarette in the past and identified themselves as a past smoker were labeled "experimenter/ex-smoker."

\subsection{E-cigarette use}

E-cigarette use was assessed in terms of lifetime e-cigarette use and past $30 \mathrm{~d}$ e-cigarette use. To assess lifetime use, participants were asked "Have you ever used electronic or e-cigarettes?" (1(yes), 2(no)). Past $30 \mathrm{~d}$ e-cigarette use was assessed with a single item on a 12-point scale: "How many times have you used e-cigarettes in the past 30 days?" For analyses purposes, past $30 \mathrm{~d}$ e-cigarette use in the present study was dichotomized into use (1) versus non-use (0).

\subsection{Data analysis}

Data were analyzed in SPSS. The relationships between independent and dependent variables were examined by testing two logistic regression models. The dependent variables were lifetime e-cigarette use and past-30 d e-cigarette use. Independent variables were demographic variables (i.e., age, gender, ethnicity), online exposure to e-cigarette information, TV exposure to ecigarette information, traditional cigarette use, friend e-cigarette use, and family e-cigarette use. For analysis purposes, ethnicity was dummy-coded with White as the reference category. 


\subsection{Limitations}

The cross-sectional study design is a limitation of this study, and due to the need to include a representative number of cigarette users, the survey data was gathered from a convenience sample. Future studies generating results based on a random, more representative sample may be more generalizable. Future research could also include longitudinal studies that examine ecigarette use behaviors and cessation rates among adolescents and young adults over time. Additionally, qualitative studies that include focus groups would allow for a broader understanding of personal motivations and intentions connected with e-cigarette use.

\section{Results}

In the current study, $44 \%$ of the participants were lifetime e-cigarette users and $28 \%$ of the participants had used e-cigarettes at least once in the past $30 \mathrm{~d}$. Traditional cigarette smokers, those individuals who smoke sometimes or every day, accounted for $35 \%$ of the study participants. Approximately $38 \%$ of the participants indicated that they were curious about ecigarettes and had looked them up online. Over half (53\%) of the respondents had at least one close friend and $26 \%$ had at least one family member who used e-cigarettes. About $19 \%$ of the students stated that they got most of their information from advertisements on television.

\subsection{Logistic regression analysis}

The table below shows the results of the logistic regression analysis. Relative to White, Filipino ethnicity was significantly associated with higher lifetime and past-30 d e-cigarette use. No similar associations were found for East Asian and NH/PI young adults. As expected, past-30 d cigarette smoking was significantly associated with both lifetime and past-30 d e-cigarette use. Online exposure to e-cigarette information was significantly associated with higher likelihood of lifetime and past-30 d e-cigarette use. However, TV exposure to e-cigarette information was not associated with e-cigarette use. Having family and friends who used e-cigarettes was positively associated with recent e-cigarette use.

Table 1. Results of the logistic regression analysis examining associations of independent variables with lifetime and pst-30 d e-cigarette use.

\begin{tabular}{|c|c|c|c|c|}
\hline & \multicolumn{2}{|c|}{ Lifetime e-cigarette use } & \multicolumn{2}{|c|}{ Past-30-day e-cigarette use } \\
\hline & OR & CI & OR & CI \\
\hline Age & $\begin{array}{l}0.9 \\
8\end{array}$ & 0.92 to 1.03 & $\begin{array}{l}0.9 \\
2\end{array}$ & 0.85 to 0.99 \\
\hline Male Gender & $\begin{array}{l}1.3 \\
3\end{array}$ & 0.74 to 2.41 & $\begin{array}{l}0.9 \\
6\end{array}$ & 0.47 to 1.95 \\
\hline East Asian & $\begin{array}{l}1.2 \\
2\end{array}$ & 0.58 to 2.61 & $\begin{array}{l}1.3 \\
6\end{array}$ & 0.51 to 3.64 \\
\hline Filipino & $\begin{array}{l}2.1 \\
5\end{array}$ & 1.05 to $4.40^{*}$ & $\begin{array}{l}2.5 \\
7\end{array}$ & 1.05 to $6.28^{*}$ \\
\hline $\begin{array}{l}\text { Native Hawaiian/Pacific } \\
\text { Islander }\end{array}$ & $\begin{array}{l}1.7 \\
1\end{array}$ & 0.75 to 3.88 & $\begin{array}{l}2.1 \\
7\end{array}$ & 0.77 to 5.94 \\
\hline $\begin{array}{l}\text { Past-30-day-cigarette } \\
\text { smoking }\end{array}$ & $\begin{array}{l}4.2 \\
1\end{array}$ & 2.18 to $8.17^{* * *}$ & $\begin{array}{l}6.0 \\
1\end{array}$ & 2.83 to $12.8^{* * *}$ \\
\hline $\begin{array}{l}\text { Online exposure to e- } \\
\text { cigarette information }\end{array}$ & $\begin{array}{l}1.4 \\
0\end{array}$ & 1.06 to $1.84^{*}$ & $\begin{array}{l}1.4 \\
7\end{array}$ & $1.08,2.00^{*}$ \\
\hline
\end{tabular}

Table 1. Continue to the next page 


\begin{tabular}{|l|l|l|l|l|}
\hline \multirow{2}{*}{} & \multicolumn{2}{|c|}{ Lifetime e-cigarette use } & \multicolumn{2}{c|}{ Past-30-day e-cigarette use } \\
\cline { 2 - 5 } & OR & CI & OR & CI \\
\hline $\begin{array}{l}\text { TV exposure to e- } \\
\text { cigarette information }\end{array}$ & 1.0 & 0.84 to 1.27 & $\begin{array}{l}0.8 \\
8\end{array}$ & 0.68 to 1.15 \\
\hline Friends e-cigarette use & 1.2 & 1.10 to $1.37^{* *}$ & $\begin{array}{l}1.3 \\
4\end{array}$ & 1.18 to $1.51^{* *}$ \\
& 3 & & $\begin{array}{l}1.6 \\
8\end{array}$ & 1.20 to $2.35^{* *}$ \\
\hline Family e-cigarette use & 1.3 & $0.96,1.79$ & & \\
& 1 & &
\end{tabular}

\section{Discussion}

This study contributes to a growing body of literature which clearly shows that e-cigarette use is a crucial health concern among adolescents and young adults. This study also demonstrates a link between college students' dual use of traditional cigarettes and e-cigarettes, which results in increased consumption of nicotine and can contribute to subsequent nicotine addiction. Current study findings show that college students who accessed e-cigarette information online were more likely to use e-cigarettes themselves. Online e-cigarette information sources are strategically designed and marketed to gain attention and entice more e-cigarette users and research suggests that the promotion of e-cigarettes through advertisements and related marketing are spreading internationally through the internet [6]. In contrast, e-cigarette information received from television did not seem to significantly influence students' ecigarette use behavior. A plausible explanation is that the adolescent and young adult population is more influenced by the internet and on-line e-cigarette information, whereas television advertisements may not be an effective marketing strategy for e-cigarettes among this population.

Previous studies have found that college students who smoke are more likely to have family members and friends who use traditional cigarettes. This study builds on these previous findings, demonstrating that having family members and friends who use e-cigarettes increases the likelihood that students will use e-cigarettes themselves. E-cigarette use behavior within a students' family or circle of friends not only normalizes the behavior by increasing tolerance towards the behavior, but also encourages others to model the behavior. As a result, if ecigarettes become more prevalent on college campuses, increased visibility of the product could lead to renormalization of smoking, and thus adversely impact social norms regarding cigarette smoking.

\section{Recommendation}

Health promotion campaigns should be developed to include e-cigarette use in campus-wide tobacco control education efforts. Educational outreach and cessation programs should also include information that specifically addresses the misperception that e-cigarettes pose minimal health risks. Counter-marketing strategies should clearly identify ways that tobacco and ecigarette companies target youth by correcting misinformation generated by the tobacco industry regarding the benefits of e-cigarettes. In addition, social norms marketing educational campaigns can be effective in influencing the social norms associated with e-cigarettes, ultimately reducing the rate of e-cigarette use on college campuses.

\subsection{Social norms marketing intervention}

Social norms theory has demonstrated that students often misperceive the frequency with which their peers engage in unhealthy behaviors and that these misperceptions have an impact on 
individual behavior $[11,12]$. Misperceptions regarding substance use such as tobacco product use can result from a number of sources including perceptions of what is considered normal on college campuses and an overall acceptance regarding the frequency of peers' risk-taking behaviors [13]. Social norms marketing is based on the central concept of social norms theory, recognizing that people's behavior is influenced by their perceptions of what is typical behavior within their community. Therefore, if social norms marketing can be used to market healthy behavioral choices and to inform people that the majority of their peers do not use tobacco products, this can potentially lead students to avoid smoking and e-cigarette use [13].

Social norms marketing is quite compatible with service-learning. Service learning provides a means of connecting students' academic study to community service which promotes active and responsible citizenship $[14,15]$. Similarly, social norms marketing focuses on promoting positive behavioral change for the good of the individual and the community [14-16]. As a result, students engaged in service learning can become the change agents who actively market and replace the misperceived health norms with actual norms on a college campus [14-16].

Service learners at the University of Hawaii are actively involved in the development, dissemination and evaluation of social norms marketing health education campaigns throughout the campus community. Recently a social norms marketing campaign focusing on e-cigarettes was developed in direct response to the findings of this study. The goal of the campaign is to market and disseminate the social norms education associated with e-cigarette use and ultimately reduce the rate of e-cigarette use at the University of Hawaii.

The University of Hawaii and many other college and universities utilize the Montana Model as a guide for developing social norms marketing campaigns on their campuses [16]. The following section provides an overview of the seven steps of the social norms marketing model and illustrates how the model can be used to address e-cigarette as well as other health and wellness issues on college campuses.

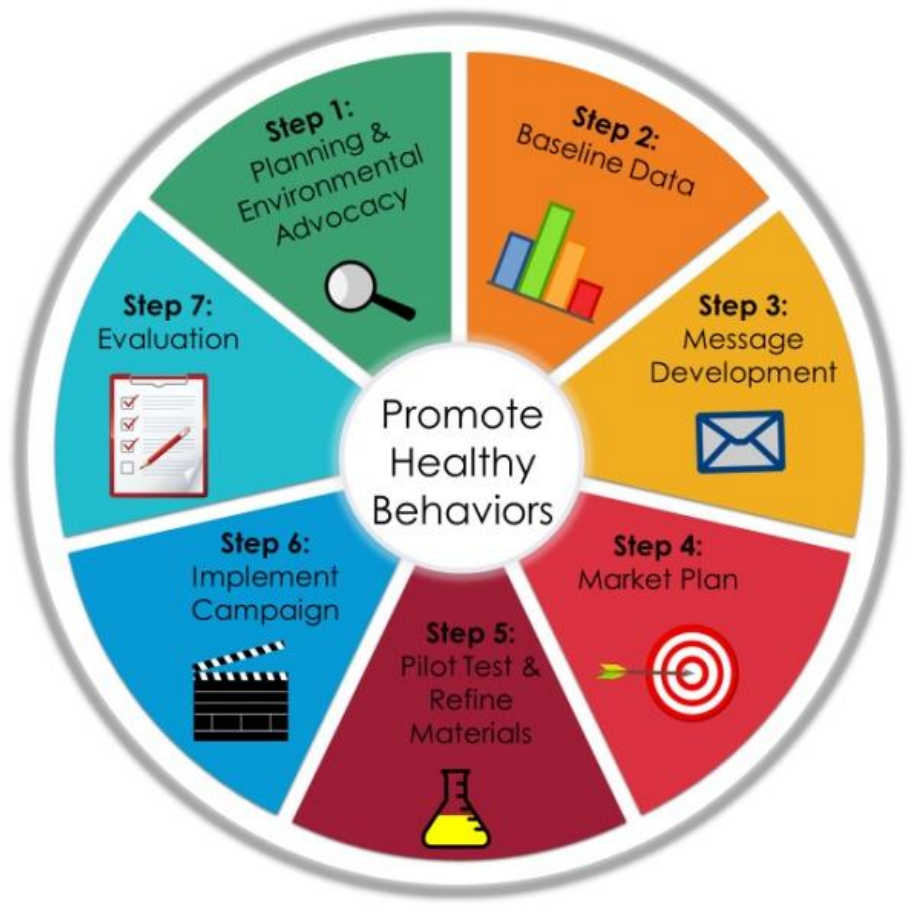

Fig. 1. Seven step model for developing social norms marketing campaigns to promote healthy behaviors. 


\subsubsection{Step 1: Planning and environmental advocacy}

Tobacco misuse continue to be major health concerns which impact health and wellness on college campuses. Service learners can design and implement social norms marketing campaigns to correct tobacco use misperceptions and encourage healthier behaviors.

\subsubsection{Step 2: Baseline data}

Data is used to establish a baseline of health behaviors on campus. The disparity between perceived behaviors and actual norms drives the social norms marketing campaign. Service learning students are involved in the data gathering and data analysis process.

\subsubsection{Step 3 and Step 4: Message development and market plan}

(i). Product - Health messages are marketed to promote a reduction of high risk behaviors and an increase in protective behaviors.

(ii). Price-Based on the desire to fit in with campus norms and one's peer group.

(iii). Promotion - Strategies for getting out social norm messages include posters, gear items, classroom presentations and health fairs, and social media. Service learning students are responsible for driving the campaign throughout the campus community.

(iv). Place-Messages are disseminated in academic buildings, residence halls, campus center, athletics. Service learners monitor the campaign on a weekly basis and become the recognized as the leaders of the campus-wide educational effort.

(v). Partners-Numerous academic departments, and student residential housing.

\subsubsection{Step 5: Pilot test and refine materials}

Service learners periodically review and provide feedback on accuracy, appeal and believability of the campaign messages. New messaging is developed throughout the school year to keep the campaign fresh and relevant.

\subsubsection{Step 6: Implement campaign}

Service learning students are responsible for saturating the campus community with the social norms poster campaign and monitoring the placement of the campaign. Service learners are also actively promoting social norms education in classroom presentations and health fairs. Service learners are teaching their peers about the true norms on campus and provide accurate information related to the health risks of e-cigarette use.

\subsubsection{Step 7: Evaluate Campaign}

Service learners conduct intercept surveys during the school year to measure saturation level and believability of the campaign. Service learners also assist with the behavioral health surveys that are used for next year's social norms marketing education campaign.

\section{Conclusion}

Social normative education campaigns are carefully designed and placed media campaigns that result in greater awareness of inter-related health and social issues. Both service learning and social norms marketing focus on a common thread, understanding and addressing the needs of 
the target community. When service learning students take an active role in social norms marketing campaigns on college campuses, they become an integral component of a campuswide prevention education strategy. The melding of service learning and social norms marketing ultimately creates long-term attitudinal and behavior change shifts on college campuses.

\section{References}

1. P. Pokhrel, M.A. Little, P. Fagan, N. Muranaka, T.A. Herzog. Addict. Behav, 39,6:10621065(2014). https://www.ncbi.nlm.nih.gov/pubmed/24630824

2. R. Chapman. State health officer's report on e-cigarettes: A community threat. California: California Department of Public Health, California Tobacco Control Program (2015). http://tobaccofreeca.com/wp-content/uploads/2016/07/State-Health-e-cig$\underline{\text { report digital.pdf }}$

3. L.O. Gostin, A.Y. Glasner, JAMA, 312,6:595-596(2014). https://www.ncbi.nlm.nih.gov/pubmed/24979277

4. P. Pokhrel, P. Fagan, L. Kehl, T.A. Herzog. Am. J. Health Behav., 39,1:121-131(2015). https://www.ncbi.nlm.nih.gov/pubmed/25290604

5. M. Cooper, A. Loukas, M.B. Harrel, C.L. Perry. Journal of American College Health, 65,2:103-111(2017). https://www.ncbi.nlm.nih.gov/pmc/articles/PMC5278646/

6. J.H. Cho, E. Shin, S. Moon. Journal of Adolescent Health, 49,5:542-546(2011). https://www.jahonline.org/article/S1054-139X(11)00273-4/fulltext

7. L.M. Dutra, S.A. Glantz, JAMA Pediatrics, 168,7:610-617(2014). https://www.ncbi.nlm.nih.gov/pubmed/24604023

8. R.A. Grana, P.M. Ling, N. Benowitz, S. Glantz, Circulation, 129,19:490-492(2014). https://www.ncbi.nlm.nih.gov/pubmed/24821830

9. J.M. Kessel. China's E-cigarette boom lacks oversight for safety. New York: New York Times (2014). https://www.nytimes.com/2014/12/14/business/international/chinas-ecigarette-boom-lacks-oversight-for-safety-.html

10. J.K. Pepper, E.L. Sherry, K.M. Ribisl, B.G Southwell, N.T Brewer. Tob. Control, 23,3:3136(2014). https://www.ncbi.nlm.nih.gov/pmc/articles/PMC4078668/

11. L.C. Lederman, L.P. Stewart. Changing the culture of college drinking: A socially situated health communication campaign. New York: Hampton Press, Cresskill, NJ (2005). https://trove.nla.gov.au/work/7410699?q\&versionId=8530522

12. K. Scholly, A.R. Katz, J. Gascoigne, P.S. Holk. J. Am. Coll. Health, 53,4:159-166(2005). https://www.ncbi.nlm.nih.gov/pubmed/15663064

13. M.P. Martens, J.C. Page, E.S. Mowry, K.S. Damann, K.K. Taylor, M.D. Cimini. J. Am. Coll. Health, 54,5:295-300(2006). https://www.ncbi.nlm.nih.gov/pubmed/16539222

14. C. Domegan, R.G. Bringle, Journal of Nonprofit \& Public Sector Marketing, 22,3:198216(2010). https://www.researchgate.net/publication/259745592_Charting the Implication_of_Social Marketing_for_Service_Learning

15. M.A. Flynn, E. Carter, Communication Education, 65,3:322-337(2016). https://www.tandfonline.com/doi/full/10.1080/03634523.2015.1107112

16. H.W. Perkins. The social norms approach to preventing school and college age substance abuse: A handbook for educators, counselors, and clinicians. San Francisco: Jossey-Bass (2003). https://www.amazon.com/Social-Approach-Preventing-College-

Substance/dp/078796459X 